\title{
Complete diagnostics and clinical approach for a female patient with unusual glioblastoma: A case study
}

\author{
FILIP SAMAL ${ }^{1 *}$, LIBOR STANEK $^{2,3 *}$, MICHAL FILIP $^{1}$, PAVEL HANINEC $^{1}$, ALES VÍCHA $^{4}$, ZDENEK MUSIL $^{4,5}$, \\ PETRA TESAROVA $^{2}$, LUBOS PETRUZELKA ${ }^{2}$, DRAHOMIRA SPRINGER $^{6}$, MILENA KRALICKOVA $^{3}$, \\ MILADA KOHOUTOVA $^{5}$ and TOMAS ZIMA ${ }^{6}$
}

${ }^{1}$ Department of Neurosurgery, Third Faculty of Medicine, Charles University and University Hospital Kralovske Vinohrady, 10034 Prague 10; ${ }^{2}$ Department of Oncology, First Faculty of Medicine, Charles University and General University Hospital, 12808 Prague 2; ${ }^{3}$ Department of Histology and Embryology, Faculty of Medicine in Pilsen, Charles University, 30166 Pilsen; ${ }^{4}$ Department of Paediatric Haematology and Oncology, Second Faculty of Medicine, Charles University, 15000 Prague 5; ${ }^{5}$ Institute of Biology and Medical Genetics; ${ }^{6}$ Institute of Medical Biochemistry and Laboratory Diagnostics, First Faculty of Medicine, Charles University and General University Hospital, 12808 Prague 2, Czech Republic

Received August 25, 2015; Accepted November 24, 2015

DOI: $10.3892 / \mathrm{mco} .2016 .891$

\begin{abstract}
The present study reports a case of a 44-year-old female patient with a large frontal lobe tumor who underwent surgery using a modern navigation system SonoWand that combines the advantages of a non-frame navigation system with intraoperative real-time ultrasound imaging. The right frontal lobe tumor consisted of two morphologically different sections. A diffuse astrocytoma grade II and a glioblastoma grade IV were identified. These tumors were relatively substantially separated. A 17 p deletion, including TP53, was detected in a diffuse astrocytoma but not in a glioblastoma. EGFR and MDM2 amplifications were detected only in a glioblastoma. Detection of these amplifications is typical for primary glioblastomas. These findings support our assumption of two independent tumors. The KRAS, BRAF and EGFR gene mutations were also detected in a glioblastoma. Such an accumulation of molecular mutations is rare in one tumor. Following oncological treatment the patient was cared for in the oncological center and survived for 15 months after the surgery without any signs of a disease. This is an unusual case, and to the best of our knowledge, is not frequently published in literature.
\end{abstract}

Correspondence to: Dr Libor Stanek, Department of Oncology, First Faculty of Medicine, Charles University and General University Hospital, U Nemocnice 2, 12808 Prague 2, Czech Republic

E-mail: stanek.libor@seznam.cz

${ }^{*}$ Contributed equally

Key words: duplicate tumor, low-grade glioma, glioblastoma multiforme, sononavigation, RAS mutation

\section{Introduction}

High-grade astrocytomas are the most difficult tumors to treat. In 2009, 412 new cases of astrocytomas were diagnosed in the Czech Republic, which accounts for 3.92 new tumors per 100,000 people. In total, $57.9 \%$ of newly diagnosed cases are glioblastomas, the most aggressive variant of astrocytomas. Two types of glioblastoma multiforme (GBM) can be recognised. Secondary GBMs develop from anaplastic or diffuse astrocytomas. Primary GBMs do not have any previous developmental phases (1).

Standard treatment includes maximal cytoreductive surgery followed by concomitant chemotherapy and adjuvant chemotherapy. Chemoradiotherapy with temozolomide following the surgery significantly extended overall survival. Median survival of patients with concomitant chemoradiotherapy was 14.6 vs. 12.1 months without chemotherapy, and 2-year survival was 26 vs. $10 \%$ without chemotherapy (1). Radicality of resection is an important prognostic factor. According to a 2001 study, the optimal resection denotes $>98 \%$ of a preoperative tumor volume. In the case of radical resection the median survival is 13 vs. 8.8 months in the case of lower radicality (2). Regardless of this comprehensive treatment, a prognosis of glioblastoma patients remains extremely poor. The addition of molecular biological examinations to the complete patient care could improve this unfavorable situation. These examinations help us to divide patients into individual prognostic groups and to choose the appropriate therapy, or they could possibly help us detect formerly unknown factors influencing the glioblastoma prognosis. From the molecular biological point of view, the types and numbers of cumulative genetic alterations are of importance. The important alterations occur in the $\mathrm{p} 53$, PTEN and EGFR genes $(3,4)$. Molecular biological changes in primary glioblastomas are different from the molecular biological changes in secondary glioblastomas $(3,4)$.

In the case of secondary glioblastomas developing from diffuse and anaplastic astrocytomas, detected mutations, such 
as the mutation or amplification of the EGFR gene, and the deletion of the PTEN gene, are found less frequently compared to the case of primary glioblastomas (3). However, the TP53 deletion is often detected (3).

Pathophysiological protein kinase and molecular cascades, particularlyp53/MDM2/p14,p16/cyclin-dependentkinase4/retinoblastoma protein 1 and epidermal growth factor receptor (EGFR)/phosphatase and tensin homolog (PTEN)/mammalian target of rapamycin (mTOR)/phosphoinositide 3-kinase/protein kinase $\mathrm{B}$, have an important role in the pathogenesis and prognosis of GBM $(5,6)$. Glioblastomas belong to the group of highly vascular tumors, and tumor elements produce large quantities of vascular endothelial growth factor (VEGF) protein. Significantly higher concentrations of VEGF protein were detected in glioblastoma tissues in comparison with anaplastic astrocytomas (grade III) and low-grade astrocytomas (grade II) (7-9). Specific molecular biological changes in primary glioblastomas are the PTEN gene deletion, loss of heterozygosity on chromosome 10, the EGFR gene mutation and amplification, and MDM2 amplification (10). The tumor suppressor PTEN gene is located on the long arm of chromosome $10(10 \mathrm{q} 23.3)$. Its role is to block the cell cycle in the $\mathrm{G}_{1}$ phase. Malfunction of the PTEN gene is connected with the progression of low-grade astrocytomas to more malignant tumors. This mutation detection is associated with a worse tumor prognosis.

The EGFR gene can be found on the short arm of chromosome 7 and it is the most frequently amplified oncogene in primary glioblastomas. EGFR is a membrane receptor for members of the EGF-family of extracellular ligands. EGFR activation stimulates intracellular protein-tyrosine kinase activity and autophosphorylation of several tyrosine (Y) residues in the $\mathrm{C}$-terminal domain occurs. The activation of signalling cascades of protein kinases mitogen-activated protein kinase [RAS and B-Raf proto-oncogene, serine/threonine kinase (BRAF)] (11) leads to DNA synthesis and cell proliferation. EGFR amplification possibly has a role in diffuse glioblastoma infiltration into surrounding tissues.

The present case report demonstrates an unusual case of two independent gliomas in a 44-year-old female patient. A diffuse astrocytoma and a primary glioblastoma were detected morphologically and by molecular biological analysis.

\section{Case report}

A 44-year-old female patient was examined in the Department of Neurosurgery (Third Faculty of Medicine, Charles University in Prague and University Hospital Kralovske Vinohrady in Prague, Prague, Czech Republic) for headaches that were prominent for 2 months. Headaches were of an increasing intensity and a magnetic resonance imaging (MRI) scan (Figs. 1 and 2) showed a large expansive process in the right frontal lobe (81x70x76 mm), with the pressure on midline brain structures and compression of the ventricular system. The MRI scan indicated a glioma. Neurological examination revealed light left-sided hemiparesis.

Extirpation of a multicomponent tumor was performed On the surface there was a component corresponding with high-grade gliomas changing quite sharply into a low-grade glioma in the central section. Tumor extirpation was performed

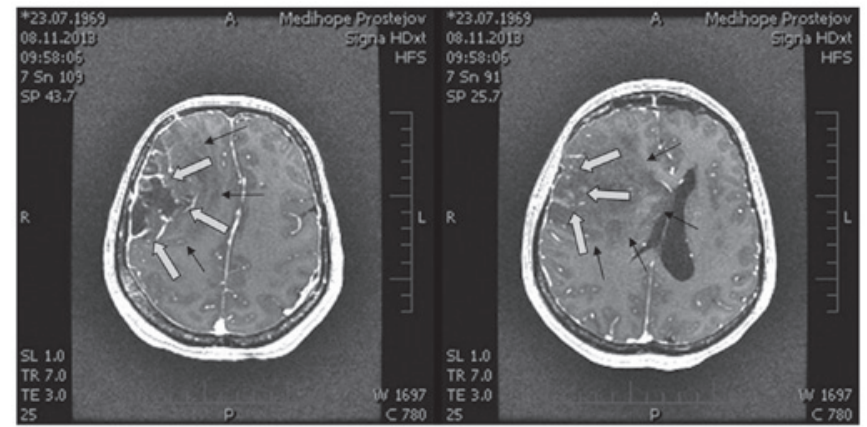

Figure 1. Preoperative magnetic resonance imaging axial scan (high- and area low-grade areas).

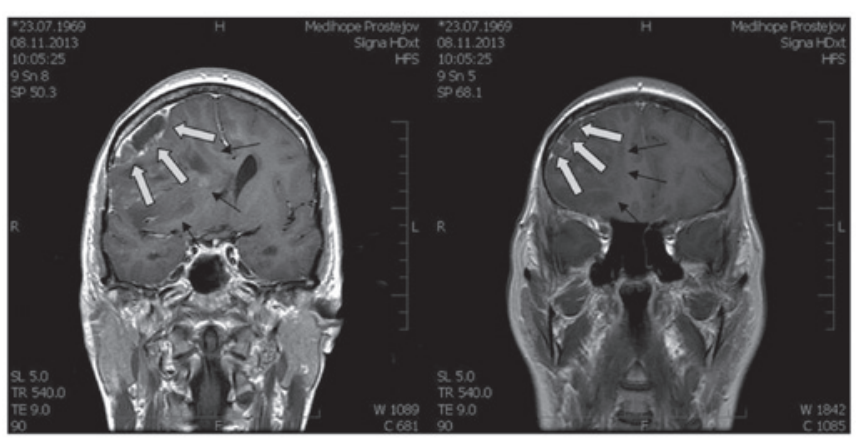

Figure 2. Preoperative magnetic resonance imaging coronal scan (high- and area low-grade areas).

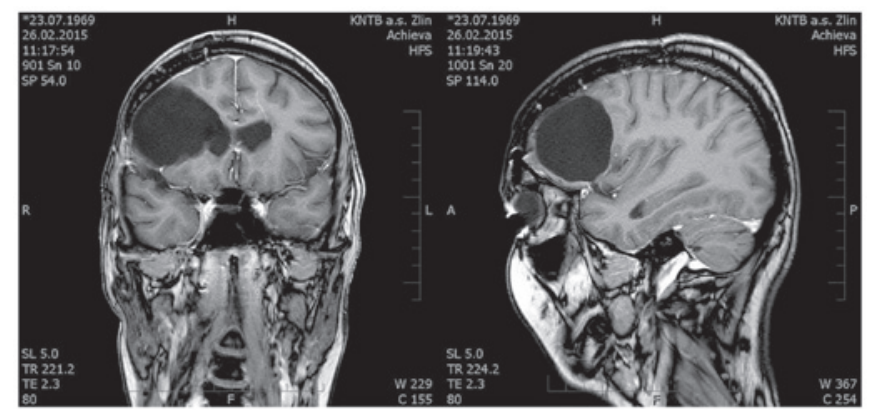

Figure 3. Postoperative magnetic resonance imaging coronar and sagital scan (high- and area low-grade areas).

using an operating microscope, navigation system SonoWand and cavitron ultrasonic surgical aspirator. The border of a low-grade component was not visible against surrounding tissues. By contrast, it was well visible in the sono image and the real-time imaging during the extirpation of this section of tumor aided with the assessment. Thus, it was possible to achieve radical resection without post-contrast enhancement in the follow-up MRI scans. Surgery was executed without any complications. Left-sided hemiparesis regressed. The tumor was removed radically, there was no evidence of disease on the follow-up MRI scans and the patient did not experience any significant complications (Fig. 3).

Samples were removed from different tumor areas for histological and molecular biological testing. The tumor component preoperatively corresponding to a low-grade glioma was pathologically identified as a diffuse astrocytoma 
Table I. Molecular profile of sample areas demonstrating the difference between low- and high-grade glioma.

\begin{tabular}{lll}
\hline Analysis & High-grade marker & Low-grade marker \\
\hline $\begin{array}{l}\text { Molecular } \\
\text { EGFR }\end{array}$ & Mut exon 18 & \\
KRAS & Mut exon 2 & WT \\
BRAF & Mut exon 15 & WT \\
FISH & & WT \\
Her2/neu & No amplification & No amplification \\
TP53 & Normal & Deletion \\
PTEN & Deletion & Normal \\
EGFR & Amplification & No amplification \\
MDM2 & Amplification & No amplification \\
IHC & & \\
p53 & Expression & No expression \\
$m T O R$ & Expression & No expression \\
\hline
\end{tabular}

FISH, fluorescence in situ hybridisation; IHC, immunohistochemistry; WT, wild-type.

with the structure of protoplasmic astrocytoma without any signs of necrosis and microvascular proliferation. In the second tumor component, preoperatively corresponding to a glioblastoma, high proliferative activity, necrosis and vascular proliferation were detected and this section of the tumor was identified as a glioblastoma.

The KRAS, BRAF and EGFR gene mutations were not detected in the areas of a low-grade tumor. TP53 deletion was detected cytogenetically, whereas other markers did not demonstrate any change (Table I). By contrast, the KRAS, BRAF and $E G F R$ gene mutations were detected in a glioblastoma. PTEN deletion and $E G F R$ and $M D M 2$ amplifications were detected cytogenetically. These findings correspond to a primary glioblastoma. Increased mRNA expression for $V E G F$ was also detected. Immunohistochemical examination showed increased p53 and $m$ TOR expression. These finding are also typical for glioblastomas. The examination results are shown in Table I.

The patient received adjuvant chemoradiotherapy with $75 \mathrm{mg} / \mathrm{m}^{2}$ temozolomide and radiation therapy 5 times weekly with 1.8-2.0 Gy/fraction (total dose of $60 \mathrm{~Gy}$ ). Following the termination of the treatment, the tiredness reported by the patient persisted without any other health difficulties. An MRI scan performed 14 months after the surgery did not show any evidence of disease.

\section{Discussion}

Occurrence of different tumor components in high-grade brain gliomas is quite common. Areas with high vascularity and necrosis, and areas showing histological changes appear. Individual tumor components are usually mixed. In the previously described tumor, the individual tumor sections representing different developmental stages were considered as macroscopically homogeneous and relatively substantially separated. These findings were confirmed histologically. The question is whether the section of the tumor corresponding to glioblastoma was developed from the already present diffuse astrocytoma or if it developed independently. On the basis of documented analyses and the accumulation of aberrations, we support the theory of tumor duplicity. In the high-grade areas, EGFR mutation was detected (12) together with the $K R A S$ and BRAF hot-spot gene changes (13). These mutations are not usually detected when the EGFR gene mutation and amplification is found. p53 mutation was also detected. p53 and $E G F R$ mutations are negative prognostic factors for survival. The detection of PTEN and $m T O R$ gene deletion that was in the high-grade areas also means a worse prognosis for patients $(14,15)$.

For the discovery of previously unknown factors that influence the prognosis of this malignant disease, it is necessary to study patients with a longer survival and unusual types of tumors from different angles, including detailed molecular genetic testing. The case reported in the present study describes an unusual case of a 44-year old patient with a glioma consisting of several morphologically different sections from low- to high-grade and showing the accumulation of genetic alterations. The prognosis of the patient is determined by the most malignant section of a tumor. At present, the patient has survived for 15 months after the surgery without any signs of radiological and clinical disease progression.

\section{Acknowledgements}

The present study was supported by the Ministry of Education of the Czech Republic (programs PRVOUK-P27/LF1/1 and PRVOUK-P25/LF1/2).

\section{References}

1. Stupp R, Mason WP, van den Bent MJ, Weller M, Fisher B, Taphoorn MJ, Belanger $\mathrm{K}$, Brandes AA, Marosi C, Bogdahn U, et al: Radiotherapy plus concomitant and adjuvant temozolomide for glioblastoma. N Engl J Med 352: 987-996, 2005.

2. Lacroix M, Abi-Said D, Fourney DR, Gokaslan ZL, Shi W, DeMonte F, Lang FF, McCutcheon IE, Hassenbusch SJ, Holland E, et al: A multivariate analysis of 416 patients with glioblastoma multiforme: Prognosis, extent of resection, and survival. J Neurosurg 95: 190-198, 2001.

3. Kim B, Myung JK, Seo JH, Park CK, Paek SH, Kim DG, Jung HW and Park SH: The clinicopathologic values of the molecules associated with the main pathogenesis of the glioblastoma. J Neurol Sci 294: 112-118, 2010.

4. Ohgaki $\mathrm{H}$ and Kleihues P: Genetic alterations and signaling pathways in the evolution of gliomas. Cancer Sci 100: 2235-2241, 2009.

5. Deng C, Zhang P, Harper JW, Elledge SJ and Leder P: Mice lacking p21CIP1/WAF1 undergo normal development, but are defective in G1 checkpoint control. Cell 82: 675-684, 1995.

6. Slampa P: Gliomy-soucasná diagnostika a lecba. Maxdorf: 97-102, 2013 (In Czech).

7. Takano S, Yoshii Y, Kondo S, Suzuki H, Maruno T, Shirai S and Nose T: Concentration of vascular endothelial growth factor in the serum and tumor tissue of brain tumor patients. Cancer Res 56: 2185-2190, 1996.

8. Shim JW, Koh YC, Ahn HK, Park YE, Hwang DY and Chi JG: Expression of bFGF and VEGF in brain astrocytoma. J Korean Med Sci 11: 149-157, 1996.

9. Oehring RD, Miletic M, Valter MM, Pietsch T, Neumann J, Fimmers R and Schlegel U: Vascular endothelial growth factor (VEGF) in astrocytic gliomas-a prognostic factor? J Neurooncol 45: 117-125, 1999.

10. Toledo F and Wahl GM: MDM2 and MDM4: p53 regulators as target in anticancer therapy. Int J Biochem Cell Biol 39: 1476-1482, 2007. 
11. Oda K, Matsuoka Y,Funahashi A and Kitano H: A comprehensive pathway map of epidermal growth factor receptor signaling. Mol Syst Biol 1: 2005.0010, 2005.

12. Verhaak RG, Hoadley KA, Purdom E, Wang V, Qi Y, Wilkerson MD, Miller CR, Ding L, Golub T, Mesirov JP, et al: Integrated genomic analysis identifies clinically relevant subtypes of glioblastoma characterized by abnormalities in PDGFRA, IDH1, EGFR, and NF1. Cancer Cell 17: 98-1101, 2010.

13. Knobbe CB, Reifenberger J and Reifenberger G: Mutation analysis of the Ras pathway genes NRAS, HRAS, KRAS and BRAF in glioblastomas. Acta Neuropathol 108: 467-470, 2004
14. Ruano Y, Ribalta T, de Lope AR, Campos-Martín Y, Fiaño C, Pérez-Magán E, Hernández-Moneo JL, Mollejo $\mathrm{M}$ and Meléndez B: Worse outcome in primary glioblastoma multiforme with concurrent epidermal growth factor receptor and p53 alteration. Am J Clin Pathol 131: 257-263, 2009.

15. Sesen J, Dahan P, Scotland SJ, Saland E, Dang VT, Lemarié A, Tyler BM, Brem H, Toulas C, Cohen-Jonathan Moyal E, et al: Metformin inhibits growth of human glioblastoma cells and enhances therapeutic response. PLoS One 10: e0123721, 2015. 